\title{
A Protocol for Human Serum Fucoxanthinol Quantitation using LC-MS/ MS System
}

\author{
Nana Mikami1*, Masashi Hosokawa², Masayuki Abe'², Kazuo Miyashita², Hitoshi Sohma ${ }^{1,3}$ \\ and Yasuo Kokai'
}

\author{
${ }^{1}$ Department of Biomedical Engineering, School of Medicine, Sapporo Medical University, Hokkaido, Japan \\ ${ }^{2}$ Faculty of Fisheries Sciences, Hokkaido University, Hokkaido, Japan \\ ${ }^{3}$ Department of Educational Development, Center for Medical Education, Sapporo Medical University, Hokkaido, Japan
}

*Corresponding author: Nana Mikami, Ph.D., Department of Biomedical Engineering, School of Medicine, Sapporo Medical University, S1 W17, Chuo-ku, Sapporo, Hokkaido 060-8556, Japan, Tel: +81-11-611-2111 (ext. 2548), Fax: +81-11-615-2315, E-mail:mikami7@sapmed.ac.jp

\begin{abstract}
Fucoxanthin, a marine carotenoid widely distributed in brown algae, is known to possess various health promoting activities including anti-obesity effects in humans, but little is known about its kinetics partly because of its low bioavailability. We have developed a simple and reproducible protocol for quantifying system of human serum fucoxanthinol, a fucoxanthin metabolite, used LC-MS/MS multiple reaction monitoring (MRM), and have applied for clinical trial to study fucoxanthin absorption. We established a MRM channel for fucoxanthinol (m/z 617.5 for Q1 and 109.0 for Q3 channel) with a standard curve of $R^{2}>0.99$ and an optimized pretreatment of human serum samples. Fucoxanthinol levels in sera obtained from subjects participating in clinical trials were then measured and a significant increase in the levels was observed at $4 \mathrm{hr}$ after a single oral dose of $22 \mathrm{mg}$ fucoxanthin using newly developed protocol. We propose that use of this protocol could enhance clinical investigation involving the bioavailability and functionality of fucoxanthinol.
\end{abstract}

\section{Keywords}

Fucoxanthin, Fucoxanthinol, Fucoxanthin intake, Human serum, LC- MS/MS, MRM, Clinical trial

\begin{abstract}
Abbreviations
APCl: Atmospheric pressure chemical ionization; Fx: Fucoxanthin; FxOH: Fucoxanthinol; HPLC:High-performanceliquid chromatography; LC: Liquid chromatography; LC-MS: Liquid chromatography-mass spectrometry; LC-MS/MS: Liquid chromatography-tandem mass spectrometry; LLOQ: The lower limit of quantification; LOD: The limit of detection; MCT: Medium-chain triglyceride; MRM: Multiple reaction monitoring; MS: Mass spectrometry; m/z: Mass-to-charge ratio; Q1: The first quadrupole; Q3: The third quadrupole.
\end{abstract}

\section{Introduction}

Fucoxanthin is a marine carotenoid widely found in edible brown algae such as wakame (Undaria pinnatifida), kombu (Laminaria japonica) and akamoku (Sargassum horneri), and contributes more than $10 \%$ of the estimated total production of carotenoids in nature [1]. Fucoxanthin exhibits numerous health benefits especially anticancer [2-5] and anti-obesity [6,7] activities as well as benefiting various other health related problems [8-10]. According to Abidov, et al. in a report, which focuses on the anti-obesity activity of fucoxanthin, oral administration of a dietary supplement capsule containing fucoxanthin induces weight loss and increases resting energy expenditure in obese women [11]. However, only the activities of fucoxanthin were assayed and the kinetics of possible metabolites were unidentified in the report. To determine the physiological relevance of these activities, it is essential not only to study the digestion and absorption of orally administered fucoxanthin but also to identify its metabolites and the effective relationship between fucoxanthin and its possible activity.

Dietaryfucoxanthin is incorporated intotheblood asfucoxanthinol after deacetylation in the digestive tract [12] (Figure 1), and at least in the liver of mice, is then metabolized to amarouciaxanthin A [13]. Several detection systems have been described previously for analyzing fucoxanthinol in vivo, including HPLC [14,15], LC-MS [16,17] and LC-MS/MS [18]. Low bioavailability of fucoxanthinol compared to other carotenoids in humans has been demonstrated $[14,15]$. Asai, et al. reported that serum fucoxanthinol concentration after wakame intake (including $6.1 \mathrm{mg}$ of fucoxanthin) was close to the lower limit of quantification by HPLC [14]. Therefore, to perform valid kinetics studies a more sensitive quantifying system for serum fucoxanthinol than exists at present is required. In addition to this approach, since the presence of highly concentrated proteins and other components in serum samples complicates analyses (e.g. causing background contamination by impurities), it is needed to remove them by improving preparation and analytical protocol to determine serum fucoxanthinol properly.

We aimed to develop a protocol for quantification of serum fucoxanthinol using liquid chromatography coupled with tandem mass spectrometry (MS/MS), which has a high sensitivity and a wide dynamic range. To apply the developed protocol to a large number of serum samples obtained from clinical trials, we also examined a washing step of LC for continuous analyses and evaluated the effects of pre-analytical factors, such as a freeze-thaw cycle on fucoxanthinol stability. We here report the kinetics in humans after a single dose of fucoxanthin to confirm the validity of applying the developed analysis to various clinical researches involving fucoxanthin intervention.

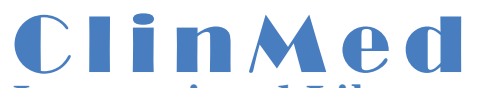
International Library

Citation: Mikami N, Hosokawa M, Abe M, Miyashita K, Sohma H, et al. (2016) A Protocol for Human Serum Fucoxanthinol Quantitation using LC-MS/MS System. J Nutri Med Diet Care 2:019 Received: July 26, 2016: Accepted: September 27, 2016: Published: September 30, 2016 Copyright: ( 2016 Mikami N, et al. This is an open-access article distributed under the terms of the Creative Commons Attribution License, which permits unrestricted use, distribution, and reproduction in any medium, provided the original author and source are credited. 


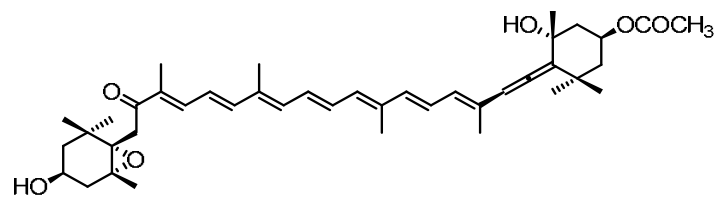

Fucoxanthin $(\mathrm{Fx}) \quad$ m/z 659.4
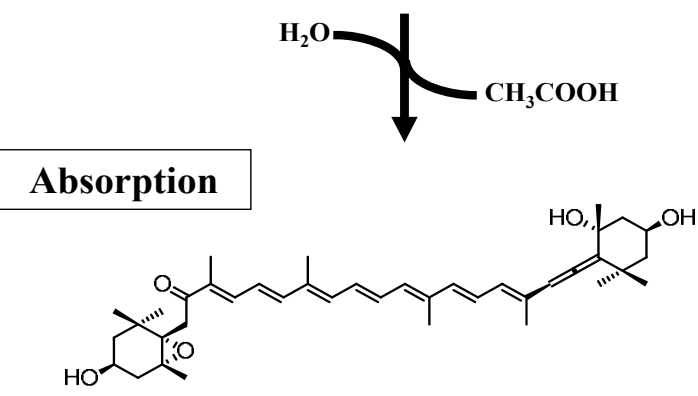

Fucoxanthinol $(\mathrm{FxOH}) \quad m / z 617.5$

Figure 1: Biotransformation of fucoxanthin to fucoxanthinol in the digestive tract. Fucoxanthin is converted to fucoxanthinol, its deacetylated form, which is absorbed from the digestive tract into the circulation (Sugawara, et al. 2002) [12].

\section{Methods}

\section{Materials}

Fucoxanthinol, fucoxanthin and solvents for LC-MS/MS analysis were purchased from Wako Pure Chemical Industries, Ltd. (Osaka, Japan), and other solvents were from Kanto Chemical (Tokyo, Japan). For clinical trials, fucoxanthin capsules were purchased from Kaneka Corporation (Osaka, Japan), prepared by Akamoku (Sargassum horneri) oil including 1\% fucoxanthin and medium-chain triglyceride (MCT) oil including lecithin and vitamin E. Contents of the capsule were as follows; containing fucoxanthin $2.2 \mathrm{mg}$ with 220 mg Akamoku oil and $20 \mathrm{mg}$ MCT oil.

\section{LC-MS/MS analysis}

The LC system consisted of an Agilent 1100 series degasser, binary pump, auto- sampler, column oven (Agilent Technologies, Santa Clara, CA, USA) and Inertsil ${ }^{\mathbb{}}$ - ODS column $(4.6 \mathrm{~mm}$ i.d. $\times 150$ $\mathrm{mm}, 3 \mu \mathrm{m}$, GL Sciences Inc., Tokyo, Japan). The HPLC mobile phases were methanol/acetonitrile $(70 / 30, \mathrm{v} / \mathrm{v})$ and elution was performed 0-5 min after sample injection ( $5 \mu \mathrm{L}$ of standard fucoxanthinol and fucoxanthin or $50 \mu \mathrm{L}$ of serum sample). The flow rate was set for 1 $\mathrm{mL} / \mathrm{min}$ at $35^{\circ} \mathrm{C}$.

The LC system was coupled with triple quadruple MS/MS 4000 QTRAP $^{\circledR}$ LC/MS/MS system (AB SCIEX, Framingham, MA, USA), which was equipped with an atmospheric pressure chemical ionization (APCI) source, and operated in positive ion mode. The MS parameters optimized were as follows: declustering potential: $76 \mathrm{~V}$; entrance potential: $10 \mathrm{~V}$; dwell time: $400 \mathrm{msec}$; curtain gas (nitrogen): $10 \mathrm{psi}$; ion source gas 1 (nitrogen): $80 \mathrm{psi}$; turbo gas temperature: $500^{\circ} \mathrm{C}$; interface heater: on; and nebulizer current: 5.0 psi. Nitrogen was used as the collision gas with a collision energy of $52 \mathrm{eV}$ and a collision cell exit potential of $7 \mathrm{~V}$. Analyst software 1.5 (AB SCIEX, Framingham, MA, USA) was used for the system control, data acquisition and data processing.

\section{Standard curve of fucoxanthinol}

Calibration standards for fucoxanthinol were prepared at 1,10 , 100,1000 , and $10000 \mathrm{ng} / \mathrm{mL}$ by successive dilution with methanol/ acetonitrile $(70 / 30, v / v)$. Five microliter of each standard was subjected to LC-MS/MS as described above and the standard curve was constructed by plotting the peak area ratio of fucoxanthinol $(y)$ versus the concentration $(\mathrm{ng} / \mathrm{mL})$ of fucoxanthinol $(x)$.
Moreover, precision and accuracy of intra- and inter-day were assessed using calibration standards of 10,100 and $1000 \mathrm{ng} / \mathrm{mL}$ (50, $500,5000 \mathrm{pg}$ on the column) fucoxanthinol and fucoxanthin. The precision and accuracy were determined by replicated analysis of 3 samples of each concentration of standard on 8 different days. Intraday analysis was performed twice a day (i.e. morning and afternoon).

\section{Extraction of fucoxanthinol from serum samples}

To quantify fucoxanthinol levels in serum samples, the serum epoxyxanthophyll fraction that included fucoxanthinol was extracted according to a method of Asai, et al. [14] with a slight modification. In brief, $1 \mathrm{~mL}$ serum, $0.2 \mathrm{~mL}$ saline, and $2 \mathrm{~mL}$ methanol including $5 \mathrm{ng}$ fucoxanthin as an internal standard were added to glass tubes and vortexed. After adding $4 \mathrm{~mL}$ dichloromethane, the mixture was centrifuged at $416 \mathrm{~g}$ for $10 \mathrm{~min}$ at room temperature. The bottom layer was collected and this extraction procedure was repeated twice. The solvent of the collected fraction was removed to dry using a centrifugal concentrator (VC-96W, Taitec, Saitama, Japan) equipped with a vacuum pump and freeze trap. The residue was dissolved in $n$-hexane/diethyl ether $(9 / 1, \mathrm{v} / \mathrm{v})$, and applied to a Bond Elut ALN (100 mg, $1 \mathrm{~mL}$ ) solid-phase extraction cartridge (Agilent Technologies, Santa Clara, CA, USA) pretreated with $1 \mathrm{~mL} n$-hexane. After the cartridge was washed with the $1 \mathrm{~mL} n$-hexane/diethyl ether $(9 / 1, \mathrm{v} / \mathrm{v})$, the epoxyxanthophylls were eluted with $1 \mathrm{~mL}$ diethyl ether/ ethanol $(4 / 1, \mathrm{v} / \mathrm{v})$. The eluate was dried in vacuo, re-dissolved in methanol/acetonitrile $(70 / 30, \mathrm{v} / \mathrm{v})$.

After these procedures described by Asai, et al., we added to ultrafiltrate with Amicon ${ }^{\circledR}$ Ultra- $0.5 \mathrm{~mL} 3 \mathrm{~K}$ devices (Merck Millipore, Billerica, MA, USA) by twice centrifugation at $14,000 \mathrm{~g}$ for $10 \mathrm{~min}$ at room temperature, due to remove proteins carried over into collected epoxyxanthophyll fraction during extraction.

The collected sample was dried and resuspended in $250 \mu \mathrm{L}$ of methanol/acetonitrile $(70 / 30, \mathrm{v} / \mathrm{v})$, and then the $50 \mu \mathrm{L}$ sample was subjected to LC-MS/MS analysis. Triplicates of each serum sample were independently extracted and analyzed.

\section{Quantification of fucoxanthinol in human sera}

Fucoxanthin was used as the internal standard and spiked in serum to quantify the fucoxanthinol level because its structure and solubility are similar to fucoxanthinol. A previous report [15] and our preliminary experiment (Supplementary Figure 1) showed that fucoxanthin was not detected in serum after its intake. Samples were next analyzed in tuned MRM channels of both fucoxanthinol and fucoxanthin simultaneously and the amount of fucoxanthinol in serum was calculated from the area counts for fucoxanthin (it was assumed that the ionization efficiency of fucoxanthin and fucoxanthinol were similar in this quantitation system). Furthermore, the mean intra-day precision of serum sample analyses including the preparation procedure was calculated by the area counts of spiked fucoxanthin in the analyses among 32 days.

From these analyses, we determined the limit of detection (LOD) and the lower limit of quantification (LLOQ) in serum sample. The LOD and the LLOQ were defined as the concentrations with signalto-noise ratios of 3 and 10, respectively [19].

\section{Effects of freeze-thaw stability on fucoxanthinol}

Application of our assay to clinical research must identify variations due to changes in handling and processing of blood samples which may affect fucoxanthinol levels. To ensure optimal results for clinical fucoxanthinol levels, it is necessary to understand conditions which determine fucoxanthinol stability. Therefore, we systematically measured the impact of pre-analytical variables, particularly, freezethaw cycles ( 0 or 1 or 2 ) on fucoxanthinol.

Briefly, fucoxanthinol at concentrations of 250, 2500 and 25000 pg was spiked into $1 \mathrm{~mL}$ serum after blood collection and separation. Samples not freeze-thawed were immediately extracted as described above. In contrast, samples with 1 or 2 cycles of freeze-thaw were frozen at $-80^{\circ} \mathrm{C}$ for 5 days or 12 days ( 5 days +7 days), respectively. After freezing 


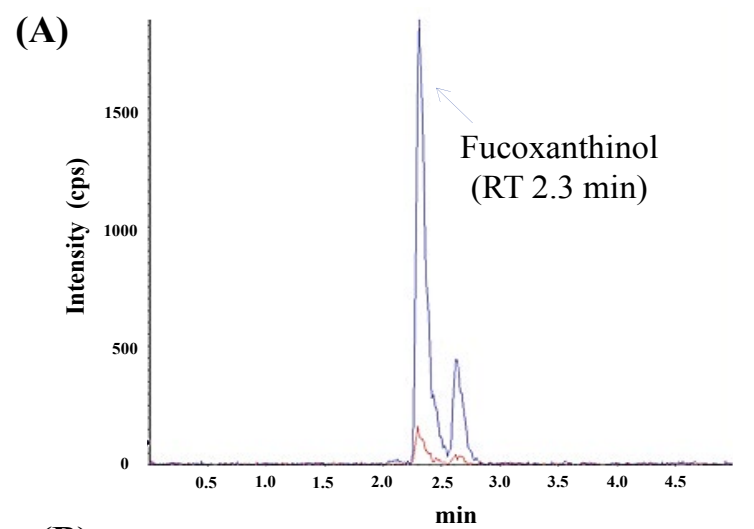

(B)

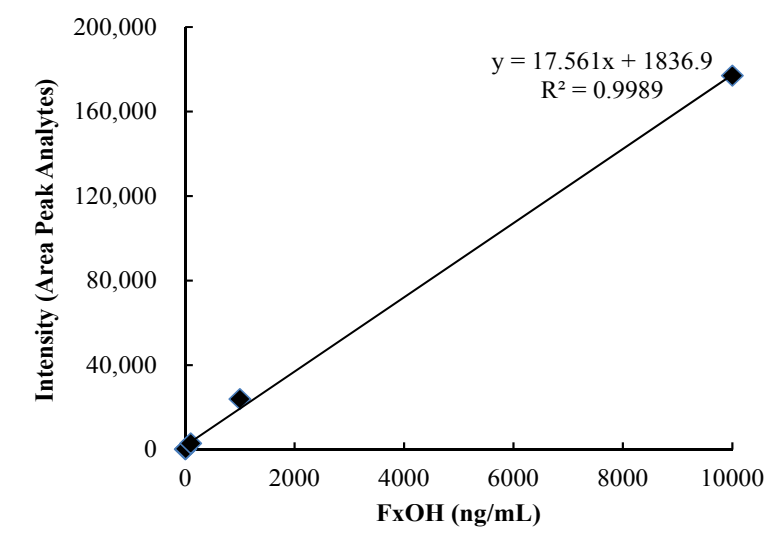

Figure 2: Detection of standard fucoxanthinol by LC-MS/MS. (A) The chromatogram of fucoxanthinol ( $1000 \mathrm{ng} / \mathrm{mL}$, i.e., $5000 \mathrm{pg}$ on column) is detected by the tuned MRM channel. The channels of fucoxanthinol (as shown in Table 1) are expressed as follows: (blue line) a quantitation ion for fucoxanthinol, m/z, 617.5-109.0; (red line) a confirmation ion for fucoxanthinol, $\mathrm{m} / \mathrm{z}, 617.5-67.0$. Fucoxanthinol was detected at $2.3 \mathrm{~min}$. The peak at $2.6 \mathrm{~min}$ might have been due to cis-isomer of fucoxanthinol [12] (B) Linearity of standard curve of fucoxanthinol detected by the tuned MRM channel using a quantitation ion for fucoxanthinol.

for each day, samples were thawed at room temperature and extracted. The $50 \mu \mathrm{L}$ of extracted sample was subjected to LC-MS/MS analysis (i.e. 50, 500 and $5000 \mathrm{pg}$ of theoretical fucoxanthinol values per inject). Triplicates of each concentration on freeze-thaw cycle were extracted and analyzed. The impact of freeze-thaw was analyzed by comparing it to fucoxanthinol on 0 cycle of freeze-thaw (expressed as 100\%).

\section{Analysis of clinical trial samples}

Subjects were 30 57 year-old healthy volunteers recruited from Sapporo Medical University (one woman and three men). The study protocols were approved by the ethical review committee of Sapporo Medical University (\#24-2-91). Written informed consent was obtained from all subjects. Throughout the study, the subjects did not change their normal diets or physical activities.

In this experiment, subjects took a single dose of $22 \mathrm{mg}$ fucoxanthin (in the form of 10 capsules). Blood was taken after 0 , 4,24 and $48 \mathrm{~h}$ of the dose and collected from a forearm vein and sat for $30 \mathrm{~min}$ in serum-separator tubes (with a coagulant). Sera were then centrifuged at $1,200 \mathrm{~g}$ for $10 \mathrm{~min}$ at room temperature and subsequently stored at $-30^{\circ} \mathrm{C}$ until analysis. After one freezethaw cycles, epoxyxanthophyll fraction was extracted in triplicate by the method described above.

\section{Statistical analysis}

Results are expressed as mean \pm SD or SE. Data comparisons were conducted using the Tukey HSD for multiple comparisons. The significance level was set at less than 0.05 . Statistical analysis was performed using JMP10 by SAS.
Table 1: Candidates of MRM channels for fucoxanthinol and fucoxanthin.

\begin{tabular}{|l|l|l|l|l|l|}
\hline $\begin{array}{l}\text { Target compound } \\
\left(\text { Exact mass, } \mathbf{a m u}^{\mathrm{b}} \text { ) }\right.\end{array}$ & Ion & $\begin{array}{l}\text { Precursor } \\
(\mathrm{Q} 1)\end{array}$ & $\begin{array}{l}\text { Product } \\
(\mathrm{Q} 3)\end{array}$ & $\begin{array}{l}\text { lon } \\
\text { intensity }\end{array}$ & $\mathrm{RT}^{\mathrm{a}}$ \\
\cline { 2 - 7 } & $\mathrm{m} / \mathrm{z}^{\mathrm{c}}$ & & $\mathrm{Cps}^{\mathrm{d}}$ & $\mathrm{min}$ \\
\hline $\begin{array}{l}\text { Fucoxanthinol } \\
(616.4127)\end{array}$ & Quantitation & 617.5 & 109.0 & 1920 & 2.3 \\
\hline Fucoxanthin & Confirmation & 617.5 & 67.0 & 1350 & 2.3 \\
\hline (658.4233) & Quantitation & 659.4 & 109.0 & 2210 & 2.7 \\
\hline & Confirmation & 659.4 & 581.3 & 1600 & 2.7 \\
\hline
\end{tabular}

Q1: the first quadrupole, Q3: the third quadrupole

a: retention time, ${ }^{b}$ : atomic mass unit, ${ }^{c}$ : mass spectrum, ${ }^{d}$ : count per second.

Table 2: Precision and accuracy of MRM channel of fucoxanthinol and fucoxanthin.

\begin{tabular}{|l|l|l|l|l|l|l|}
\hline & \multicolumn{4}{|l|}{ Fucoxanthinol } & \multicolumn{3}{l|}{ Fucoxanthin } \\
\hline ng/ml & 10 & 100 & 1000 & 10 & 100 & 1000 \\
\hline pg on column & 50 & 500 & 5000 & 50 & 500 & 5000 \\
\hline Intra-day mean (pg on column) & 39.0 & 577.0 & 4972.0 & 50.9 & 500.3 & 5002.4 \\
\hline Precision (\%CVa) & 21.2 & 2.7 & 2.0 & 16.9 & 11.8 & 6.2 \\
\hline Accuracy (\%) & 78.6 & 115.3 & 99.4 & 101.8 & 100.7 & 100.0 \\
\hline Inter-day mean (pg on column) & 41.0 & 543.0 & 4969.0 & 47.8 & 502.5 & 4999.8 \\
\hline Precision (\%CV) & 21.7 & 6.6 & 3.5 & 10.0 & 3.7 & 1.8 \\
\hline Accuracy (\%) & 81.8 & 108.6 & 99.4 & 95.5 & 100.5 & 100.0 \\
\hline
\end{tabular}

a: percent coefficient of variation, b: percent ratio of measured amu per theoretical amu.

\section{Results and Discussion}

\section{MRM channel and standard curve of fucoxanthinol}

To obtain greater sensitivity for quantifying serum fucoxanthinol, we employed multiple reaction monitoring (MRM) [20]. Because the most intense signals for fucoxanthinol and fucoxanthin were observed in the first quadrupole (Q1) and the third quadrupole (Q3) masses (Table 1) during continuous infusion of standard solution of each compound at $1 \mu \mathrm{g} / \mathrm{mL}$, we selected the channel for fucoxanthinol $(\mathrm{m} / z$ $617.5,109.0)$ and for fucoxanthin $(\mathrm{m} / z 659.4,109.0)$ as quantitation ions (Table 1), respectively. We confirmed standard fucoxanthinol was detected in $\mathrm{m} / \mathrm{z} 617.5 \rightarrow 109.0$ at RT $2.3 \mathrm{~min}$ (Figure 2A). The channel was linear in the concentration range tested of $10-10000 \mathrm{ng} /$ $\mathrm{mL}$ (50-50000 pg on the column) with a correlation coefficient of 0.9989 (Figure 2B).

\section{Precision and accuracy of standard analyses}

Table 2 shows the results for precision and accuracy analyzed twice in one day or on 8 different days. The intra- and inter-day precisions in fucoxanthinol and fucoxanthin were shown in table 2. Generally, it is recommended that precision should be $<20 \%$ and accuracy between $80-120 \%$ of the theoretical value. However, in the analysis of $10 \mathrm{ng} / \mathrm{mL}$ (50 pg on the column) of fucoxanthinol, both precision and accuracy were decreased in both intra- and inter-day sample analysis. On the other hand, for the concentrations of 100 and $1000 \mathrm{ng} / \mathrm{mL}$ (500 pg and $5000 \mathrm{pg}$ on the column) of fucoxanthinol, the precision was $<7 \%$ and the accuracy was $99.4-115.3 \%$. Moreover, in all the concentrations of fucoxanthin, the precision was $<17 \%$ and the accuracy was $95.5-101.8 \%$.

\section{Examination of LC column washing step for continuous analyses of serum samples}

To remove impurities from serum and shorten the total run time, we examined the effects of washing with dichloromethane and a reconditioning step from RT $5 \mathrm{~min}$ to $60 \mathrm{~min}$. As shown in figure 3, peaks detected in MRM channels of fucoxanthinol (blue line; $m / z 617.5,109.0$, red line; $m / z 617.5,67.0$ ) were observed at least until 320 min under no washing step conditions. This was dramatically improved by washing with dichloromethane from 5 to $50 \mathrm{~min}$. Figure 3B shows no peaks after $52 \mathrm{~min}$. We thus successfully removed impurities and reduced total run time to less than $60 \mathrm{~min}$. The final washing and reconditioning steps were as follows; solvent $\mathrm{A}$ (methanol/acetonitrile, 70/30, v/v) and solvent B (dichloromethane); 


\section{(A)}

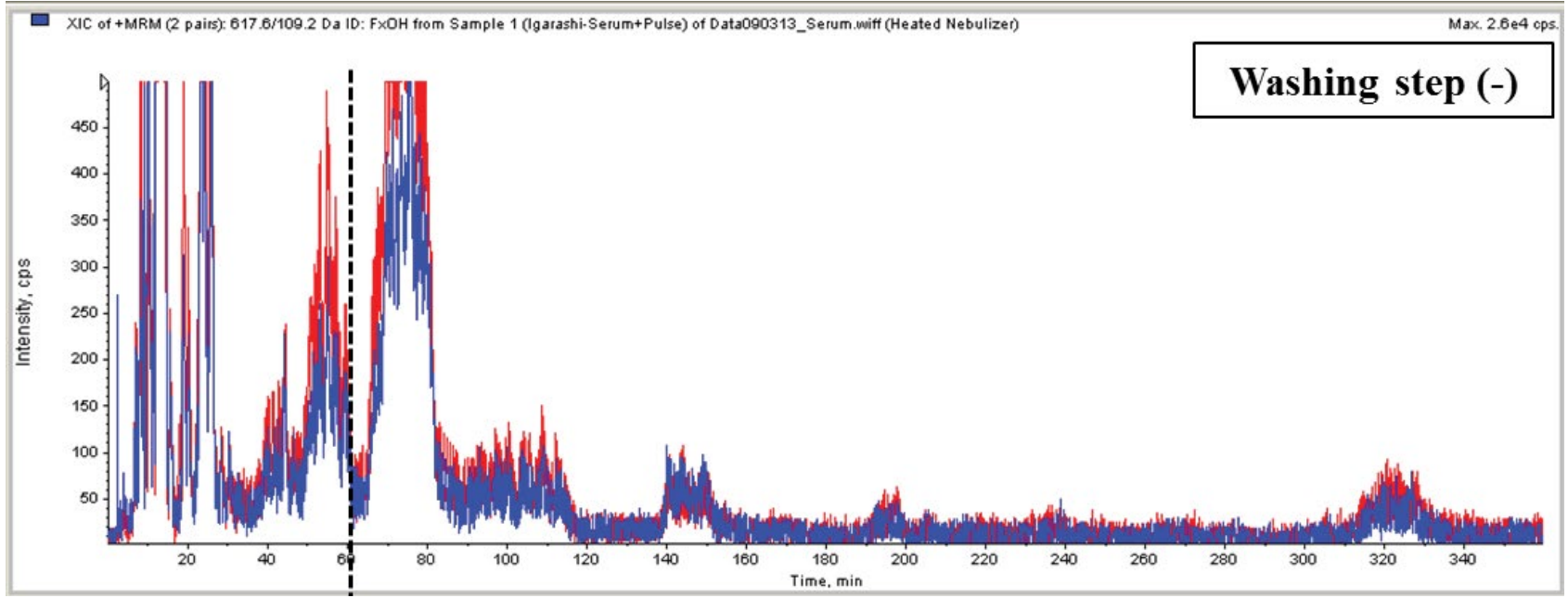

(B)

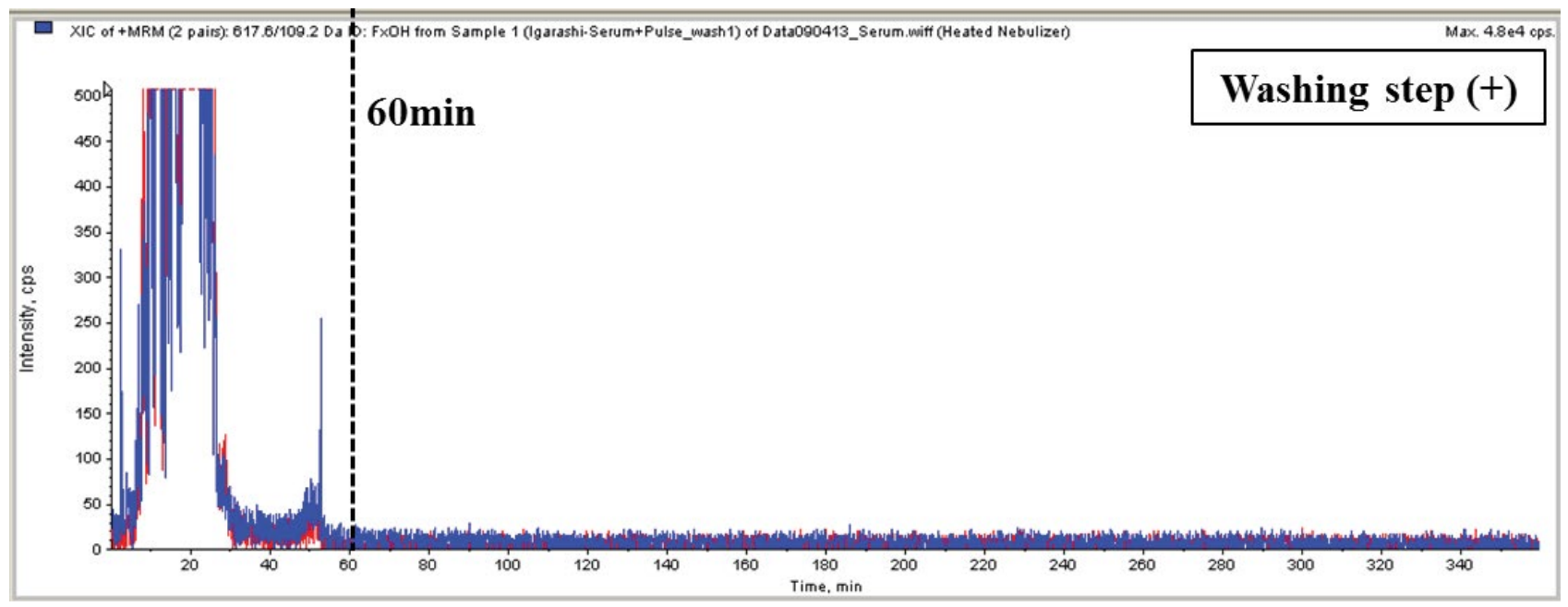

Figure 3: Examination of LC washing and reconditioning step for continuous analysis. Chromatograms were for analysis of serum sample (A) without and (B) with the washing step. Peaks detected in MRM channels of fucoxanthinol (blue line; $\mathrm{m} / \mathrm{z} 617.5,109.0$, red line; $\mathrm{m} / \mathrm{z} 617.5,67.0$ ) were observed at least until $320 \mathrm{~min}$ under no washing step condition (A). In contrast, washing with dichloromethane performed from 5 to 50 min (B) removed impurities after 60 min.

Table 3: The validation of fucoxanthinol and fucoxanthin in serum sample analyses.

\begin{tabular}{|l|l|l|}
\hline & Fucoxanthinol & Fucoxanthin \\
\hline Precision (\%CV) & 9.2 & 19.2 \\
\hline Recovery (\%) & 136.6 & 117.3 \\
\hline LOD (pg on column) & 71.1 & 36.2 \\
\hline LLOQ (pg on column) & 234.6 & 119.5 \\
\hline LOD as serum sample & $0.58 \mathrm{nM}$ & $0.27 \mathrm{nM}$ \\
\hline LLOQ as serum sample* $^{*}$ & $1.91 \mathrm{nM}$ & $0.91 \mathrm{nM}$ \\
\hline
\end{tabular}

LOD: the limit of detection, LLOQ: the lower limit of quantification

*Following the developed protocol in current study.

0-5 min with $0 \%$ B; 5-25 min with 0-100\% B with a linear gradient of B; $25-50$ min with $100 \% \mathrm{~B}$ and $50-60$ min of $0 \%$ B. By adding the washing steps, we have developed a protocol (Figure 4) and achieved continuous analyses of serum fucoxanthinol in this study without any trouble such as a clogging of LC column. Although Zhang, et al. have reported the development of the system quantifying plasma fucoxanthinol in rats by LC-MS/MS, they did not mention washing conditions of LC column in continuous sample analyses [18]. Therefore, our examined washing protocol would provide the useful information for reasonable sample analyses.

\section{Precision of serum sample analyses}

Using the developed protocol, we analyzed clinical sample. The validation of fucoxanthinol and fucoxanthin in serum sample was shown in table 3. The mean CV of peak area of spiked fucoxanthinol and fucoxanthin were calculated as $9.2 \%$ and $19.2 \%$, respectively. It indicated that this protocol kept good precision throughout serum sample preparations and analyses. In the recovery of fucoxanthinol and fucoxanthin, they were over $100 \%$ (Table 3). It may be due to the effect of serum contents. The LOD and LLOQ was fucoxanthinol and fucoxanthin from serum sample were $71.1 \mathrm{pg}$ and $234.6 \mathrm{pg}$ (in fucoxanthinol, respectively), and $36.2 \mathrm{pg}$ and $119.5 \mathrm{pg}$ (in fucoxanthin, respectively).

\section{Increase of serum fucoxanthinol levels after fucoxanthin intake}

After a single dose of $22 \mathrm{mg}$ fucoxanthin, its metabolite, fucoxanthinol, was measured in human sera at 0, 4, 24 and $48 \mathrm{~h}$. No peak representing fucoxanthinol was detected in the MRM chromatogram at $0 \mathrm{~h}$, while a rapid increase was observed at $4 \mathrm{~h}$. Peaks declined at 24 to $48 \mathrm{~h}$ (Figure 5A). Fucoxanthinol was quantified using fucoxanthin as an internal control. Serum fucoxanthinol levels were markedly elevated at $4 \mathrm{~h}$ in all subjects and were gradually decreasing in $24 \mathrm{~h}$ to $48 \mathrm{~h}$ (Figure 5B). However, the levels did not return to the baseline of $0 \mathrm{~h}(0 \mathrm{~h}, 0.4 \pm 0.2 \mathrm{nM} ; 4 \mathrm{~h}, 44.2 \pm 14.9 \mathrm{nM} ; 24 \mathrm{~h}, 6.2 \pm$ $0.9 \mathrm{nM}$; and $48 \mathrm{~h}, 1.6 \pm 0.4 \mathrm{nM}$ ), suggesting that orally administered fucoxanthin is absorbed as fucoxanthinol within $4 \mathrm{~h}$ and is not metabolized completely even after $48 \mathrm{~h}$. 


\section{A protocol for human serum fucoxanthinol quantitation}

\section{Preparation}

\section{Deproteination}

1. Add $1 \mathrm{~mL}$ of human serum sample to test tube

2. Add $0.2 \mathrm{~mL}$ of saline and $2 \mathrm{~mL}$ of methanol with $5 \mathrm{ng}$ of fucoxanthin

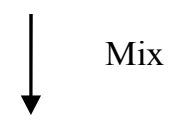

Lipid extraction

3. Add $4 \mathrm{~mL}$ of dichloromethane

4. Centrifuge the mixture at $416 \mathrm{~g}$ for $10 \mathrm{~min}$

5. Collect the bottom layer (lipid fraction) and repeat this extraction procedure twice

$\downarrow$ Dry the lipid fraction in vacuo

Separation of xanthophylls

6. Pretreat solid-phase extraction cartridge with $1 \mathrm{~mL}$ of $n$-hexane

7. Apply residue dissolved in solvent 1

8. Wash with $1 \mathrm{~mL}$ of solvent 1

Solvent 1: $n$-hexane/diethyl ether $(9 / 1, \mathrm{v} / \mathrm{v})$ Solvent 2: diethyl ether/ethanol $(4 / 1, \mathrm{v} / \mathrm{v})$

9. Elute xanthophylls with $1 \mathrm{~mL}$ of solvent 2

Dry the xanthophylls in vacuo

\section{Removal of obstacles}

10. Dissolve in methanol/acetonitrile $(7 / 3, \mathrm{v} / \mathrm{v})$

11. Ultra-centrifuge twice at $14,000 \mathrm{~g}$ for $10 \mathrm{~min}$

12. Dry the xanthophylls in vacuo and dissolve in $250 \mu \mathrm{L}$ of methanol/acetonitrile $(7 / 3, \mathrm{v} / \mathrm{v})$

Analysis

Inject $50 \mu \mathrm{L}$ for LC-MS/MS analysis

- MS Ionization: APCI source

- Ion mode: positive

- MRM channels: fucoxanthinol $(\mathrm{m} / \mathrm{z} 617.5,109.0)$, fucoxanthin $(\mathrm{m} / \mathrm{z} 659.4,109.0)$

- LC mobile phases:

A; methanol/acetonitrile $(70 / 30, \mathrm{v} / \mathrm{v}), \mathrm{B}$; dichloromethane,

$0-5 \min (0 \% \mathrm{~B}), 5-25 \min (0-100 \% \mathrm{~B}), 25-50 \min (100 \% \mathrm{~B}), 50-60 \min (0 \% \mathrm{~B})$

- Flow rate: $1 \mathrm{~mL} / \mathrm{min}$

- Retention time: fucoxanthinol (2.3 min), fucoxanthin (2.7 min)

Figure 4: A protocol for human serum fucoxanthinol quantitation.

Impact of freeze-thaw cycles on fucoxanthinol stability in human sera

To prepare for handling freeze-thawed blood samples in clinical research, we examined the impact of freeze-thaw cycles by spiking standard fucoxanthinol. The 0 cycle of adjusted recovery of fucoxanthinol was expressed as $100 \%$. As shown in figure 6 , in serum samples of $5000 \mathrm{pg}$, the recovery of fucoxanthin was significantly 


\section{(A)}
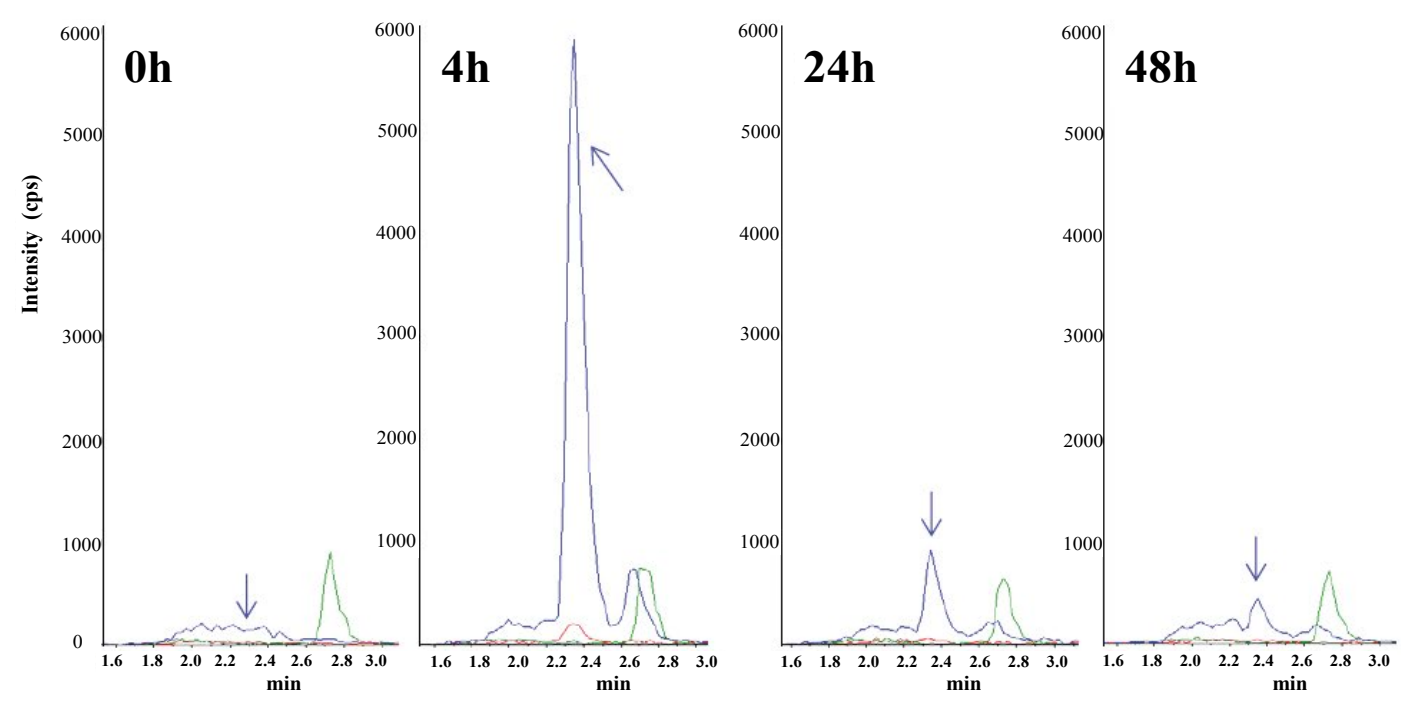

(B)

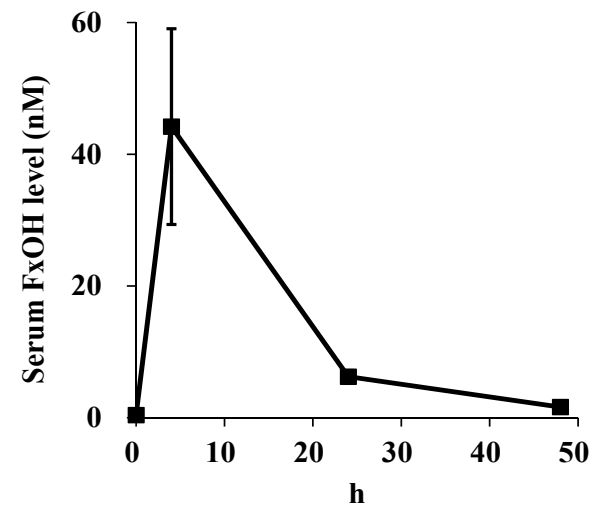

Figure 5: Changes in fucoxanthinol levels in human sera after a single $22 \mathrm{mg}$ oral dose of fucoxanthin.

(A) Chromatograms of fucoxanthinol in serum at 0, 4, 24 and $48 \mathrm{~h}$ after a single dose of oral $22 \mathrm{mg}$ fucoxanthin. In each instance, MRM channels of fucoxanthinol and fucoxanthin (as shown in table 1) are expressed as follows: (blue line) a quantitation ion for fucoxanthinol, $\mathrm{m} / \mathrm{z}, 617.5-109.0 ;$; (red line) a confirmation ion for fucoxanthinol, m/z, 617.5-67.0; (green line) a quantitation ion for fucoxanthin, m/z, 659.4-109.0; (gray line) a confirmation ion for fucoxanthin, m/z, 659.4-581.3. Peaks of fucoxanthinol and fucoxanthin were found at 2.3 and 2.7 min, respectively; (B) Serum fucoxanthinol levels were determined at $0,4,24$ and $48 \mathrm{~h}$ after a dose of $22 \mathrm{mg}$ fucoxanthin in 4 subjects. Data are mean \pm SE $(n=4)$.

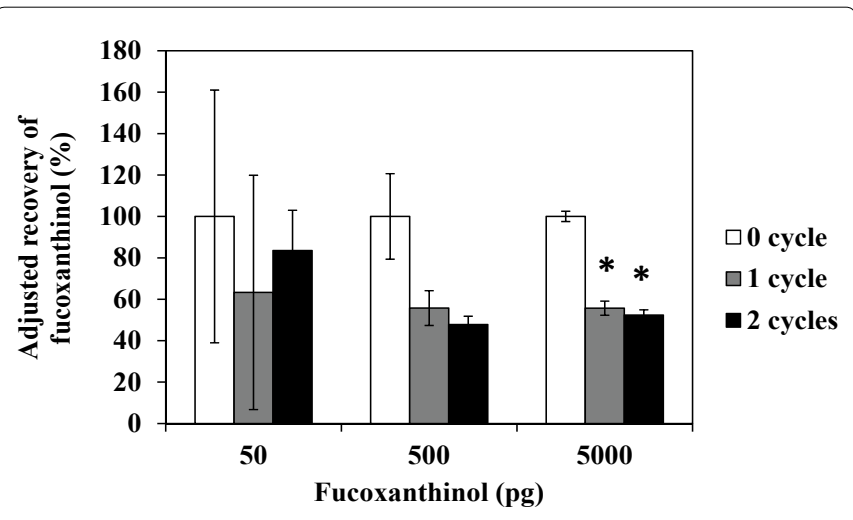

Figure 6: Impact of freeze-thaw cycle on stability of fucoxanthinol in human sera. After blood collection, fucoxanthinol $(250,2500$ and $25000 \mathrm{pg})$ was spiked in $1 \mathrm{~mL}$ of serum and freeze-thawed 0 or 1 or 2 cycles. Recovery of fucoxanthinol in sample without freeze-thaw cycle was expressed as $100 \%$ and compared to freeze-thawed samples. Data are mean $\pm \operatorname{SD}(n=3)$.

*Significantly different from 0 cycle of each concentration at $P<0.05$ by Tukey's HSD.

decreased by freeze-thaw. Moreover, it seemed that 1 or 2 freezethaw cycles tended to decrease in each concentration, although a significant difference was not observed due to the wide SD of the 0 cycle sample. Previous reports indicate that retinol, alpha-tocopherol, trans- lycopene, and trans-beta-carotene in reconstituted lyophilized serum stored at $-20^{\circ} \mathrm{C}$ were stable for at least 3 days with minimal $(<$ 5) freeze/thaw cycles [21], however, fucoxanthinol in serum samples was less stable during freeze-thawing in our study.

These results showed that repeated cycles of freeze-thaw should be avoided for fucoxanthinol quantification. It is impractical in clinical experiments to keep collected blood samples fresh until analysis. Unified freeze-thaw cycles would be important for analysis of a series of clinical samples.

\section{Conclusions}

We have developed a quantification protocol for fucoxanthinol in human sera as detected by LC-MS/MS and observed a change of fucoxanthinol levels after a single dose fucoxanthin. In this protocol, we have achieved continuous analyses of serum fucoxanthinol in this study without any trouble. These results may be applied to clinical investigations to elucidate the biological activity of fucoxanthin in humans.

\section{Acknowledgements}

This work was supported by a National Project for the Formation of Tohoku Marine Science Center (Innovation Strategy for the Industrial Seaweed Utilization Supporting Regional Seeds and Local Features of Sanriku Coast) from MEXT (Ministry of Education, Culture, Sports, Science, and Technology of Japan) and the Japan Society for the Promotion of Science Grants-in-Aid (JSPS KAKENHI Grant Number JP16K21251). 
We would like to express our gratitude to Mr. Ryo Susukida for his technical support.

\section{Ethical Statement}

This study was conducted according to the guidelines of the Declaration of Helsinki, and our study protocol and all procedures involving human subjects were approved by the ethical review committee of Sapporo Medical University (\#24-2-91).

\section{Competing Interests}

The authors have no conflict of interests.

\section{Author's Contribution}

NM designed the research and performed experiments, analyzed and interpreted the data and wrote the manuscript. $\mathrm{MH}$ and MA designed the research, especially LC-MS/MS analysis, interpreted the data and revised the manuscript. KM formulated the study conception, interpreted the data and revised the manuscript. HS interpreted the data and wrote the manuscript. YK supervised the work, designed the research, interpreted data and wrote the manuscript. All authors read and approved the final manuscript.

\section{References}

1. Dembitsky VM, Maoka T (2007) Allenic and cumulenic lipids. Prog Lipid Res 46: 328-375.

2. Hosokawa M, Kudo M, Maeda H, Kohno H, Tanaka T, et al. (2004) Fucoxanthin induces apoptosis and enhances the antiproliferative effect of the PPARgamma ligand, troglitazone, on colon cancer cells. Biochim Biophys Acta 1675: 113-119.

3. Kotake-Nara E, Terasaki M, Nagao A (2005) Characterization of apoptosis induced by fucoxanthin in human promyelocytic leukemia cells. Biosci Biotechnol Biochem 69: 224-227.

4. Kotake-Nara E, Kushiro M, Zhang H, Sugawara T, Miyashita K, et al. (2001) Carotenoids affect proliferation of human prostate cancer cells. J Nutr 131: 3303-3306.

5. Wang J, Chen S, Xu S, Yu X, Ma D, et al. (2012) In vivo induction of apoptosis by fucoxanthin, a marine carotenoid, associated with down-regulating STAT3/ EGFR signaling in sarcoma 180 (S180) xenografts-bearing mice. Mar Drugs 10: $2055-2068$

6. Maeda H, Hosokawa M, Sashima T, Funayama K, Miyashita K (2005) Fucoxanthin from edible seaweed, Undaria pinnatifida, shows antiobesity effect through UCP1 expression in white adipose tissues. Biochem Biophys Res Commun 332: 392-397.

7. Woo MN, Jeon SM, Shin YC, Lee MK, Kang MA, et al. (2009) Anti-obese property of fucoxanthin is partly mediated by altering lipid-regulating enzymes and uncoupling proteins of visceral adipose tissue in mice. Mol Nutr Food Res 53: 1603-1611.
8. Gong D, Chu W, Jiang L, Geng C, Li J, et al. (2014) Effect of fucoxanthin alone and in combination with D-glucosamine hydrochloride on carrageenan/ kaolin-induced experimental arthritis in rats. Phytother Res 7: 1054-1063.

9. Kim KN, Heo SJ, Yoon WJ, Kang SM, Ahn G, et al. (2010) Fucoxanthin inhibits the inflammatory response by suppressing the activation of NF-KB and MAPKs in lipopolysaccharide-induced RAW 264.7 macrophages. Eur J Pharmacol 649: 369-375.

10. Zheng J, Piao MJ, Keum YS, Kim HS, Hyun JW (2013) Fucoxanthin protects cultured human keratinocytes against oxidative stress by blocking free radicals and inhibiting apoptosis. Biomol Ther (Seoul) 21: 270-276.

11. Abidov M, Ramazanov Z, Seifulla R, Grachev S (2010) The effects of Xanthigen in the weight management of obese premenopausal women with non-alcoholic fatty liver disease and normal liver fat. Diabetes Obes Metab 12: $72-81$.

12. Sugawara T, Baskaran V, Tsuzuki W, Nagao A (2002) Brown algae fucoxanthin is hydrolyzed to fucoxanthinol during absorption by Caco- 2 human intestinal cells and mice. J Nutr 132: 946-951.

13. Asai A, Sugawara T, Ono H, Nagao A (2004) Biotransformation of fucoxanthinol into amarouciaxanthin $A$ in mice and HepG2 cells: formation and cytotoxicity of fucoxanthin metabolites. Drug Metab Dispos 32: 205-211.

14. Asai A, Yonekura L, Nagao A (2008) Low bioavailability of dietary epoxyxanthophylls in humans. Br J Nutr 100: 273-277.

15. Hashimoto T, Ozaki Y, Mizuno M, Yoshida M, Nishitani Y, et al. (2012) Pharmacokinetics of fucoxanthinol in human plasma after the ora administration of kombu extract. Br J Nutr 107: 1566-1569.

16. Matsumoto M, Hosokawa M, Matsukawa N, Hagio M, Shinoki A, et al. (2010) Suppressive effects of the marine carotenoids, fucoxanthin and fucoxanthino on triglyceride absorption in lymph duct-cannulated rats. Eur J Nutr 49: 243249.

17. Sangeetha RK, Bhaskar N, Divakar S, Baskaran V (2010) Bioavailability and metabolism of fucoxanthin in rats: structural characterization of metabolites by LC-MS (APCI). Mol Cell Biochem 333: 299-310.

18. Zhang $\mathrm{Y}$, Wu H, Wen $\mathrm{H}$, Fang $\mathrm{H}$, Hong Z, et al. (2015) Simultaneous determination of fucoxanthin and its deacetylated metabolite fucoxanthinol in rat plasma by liquid chromatography-tandem mass spectrometry. Mar Drugs 13: $6521-6536$

19. Vial J, Jardy A (1999) Experimental comparison of the different approaches to estimate LOD and LOQ of an HPLC method. Anal Chem 71: 2672-2677.

20. Hammad LA, Neely M, Bridge B, Mechref Y (2009) Fast liquid chromatography separation and multiple-reaction monitoring mass spectrometric detection of neurotransmitters. J Sep Sci 32: 2369-2376.

21. Brown Thomas J, Duewer DL, Kline MC, Sharpless KE (1998) The stability of retinol, alpha-tocopherol, trans-lycopene, and trans-beta-carotene in liquidfrozen and lyophilized serum. Clin Chim Acta 276: 75-87. 


\section{(A) Mixture of Fx and FxOH standard}

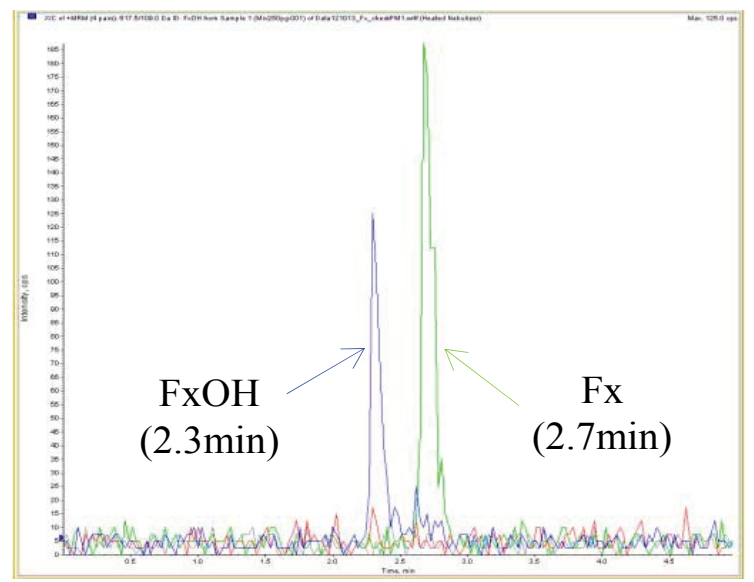

(B) Serum sample after $\mathrm{Fx}$ administation

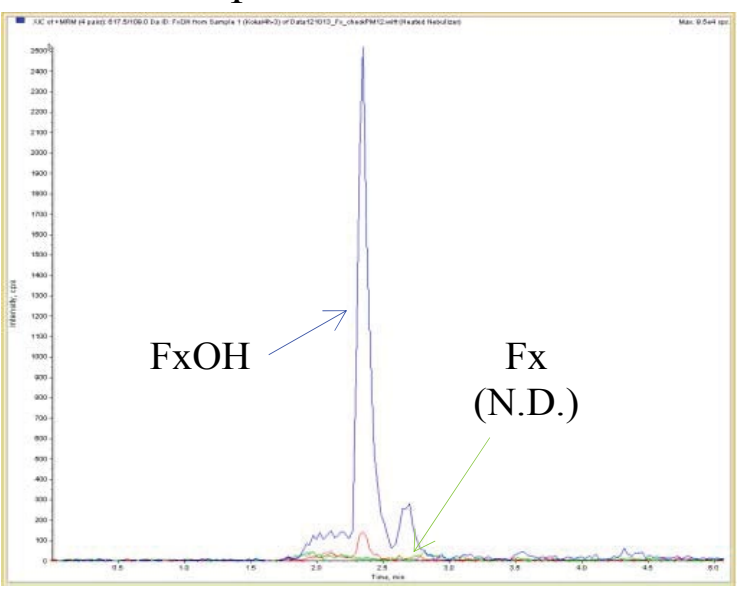

Supplementary Figure 1: Fucoxanthin was not detected in serum after its administration. After 4 hours of $22 \mathrm{mg}$ of fucoxanthin intake, we analyzed fucoxanthin and fucoxanthinol in human serum by LC-MS/MS system. The peak of fucoxanthinol was detected at $2.3 \mathrm{~min}$, that of fucoxanthin was not at $2.7 \mathrm{~min}$. (A) Mixed solution of fucoxanthin and fucoxanthinol standard; (B) Serum sample after 4 hours of fucoxanthin administration. 